\title{
Increasing Robustness of SAT-based Delay Test Generation using Efficient Dynamic Learning Techniques
}

\author{
Stephan Eggersglüß Rolf Drechsler \\ Institute of Computer Science, University of Bremen \\ Bibliothekstr. 1, 28359 Bremen, Germany \\ \{segg,drechsle\}@informatik.uni-bremen.de
}

\begin{abstract}
Due to the increased speed in modern designs, testing for delay faults has become an important issue in the postproduction test of manufactured chips. A high fault coverage is needed to guarantee the correct temporal behavior. Today's ATPG algorithms have difficulties to reach the desired fault coverage due to the high complexity of modern designs. In this paper, we describe how to efficiently integrate the reuse of learned information into state-of-the-art SATbased ATPG algorithms and, by this, reduce the number of unclassified faults significantly. For further reduction, a post-classification phase is presented. Experimental results for ATPG for delay faults on large industrial circuits show the robustness and feasibility of the approach.
\end{abstract}

\section{Introduction}

An important task in the design flow is the post-production test of each manufactured chip. Test patterns generated by Automatic Test Pattern Generation (ATPG) algorithms are applied to ensure that no erroneous chip is delivered to customers. However, classical ATPG algorithms like FAN [1] or SOCRATES [2] have difficulties to cope with the increased complexity of modern circuits. A high fault coverage is needed - in particular for designs in safety-critical application, e.g. in the field of automotive electronics.

Moreover, due to the increasing speed in modern chip design, testing of delay faults becomes more and more important. For delay testing, at least two time frames have to be considered. ATPG for delay fault models is computationally more complex than e.g. ATPG for stuck-at faults. Therefore, it needs generally more run time and results in a larger number of unclassified faults, i.e. the fault coverage is typically lower than e.g. the fault coverage for stuck-at faults.

Recently, ATPG algorithms based on Boolean Satisfiability (SAT) [3]-[6] turned out to be an efficient complement to existing classical ATPG algorithms. SAT-based algorithms do not work on a netlist but on a Boolean formula represented in Conjunctive Normal Form (CNF). Due to the homogeneity of the CNF, efficient techniques such as Boolean Constraint Propagation (BCP) [7], nonchronological backtracking and conflict-based learning [8] can be applied to speed up the search. Especially due to the use of conflict clauses, SAT-based ATPG algorithms are very robust for hard-to-test faults and can classify many faults for which classical ATPG algorithms cannot find a solution in reasonable time [6]. However, many faults still remain unclassified during delay test generation.

\subsection{Previous Work}

A promising concept to reduce the number of unclassified faults is the reuse of pervasive conflict clauses which was introduced for ATPG for stuck-at faults in [4]. Pervasive conflict clauses depend only on the circuit's function and are fault independent. In [4], only the plain concept was introduced and no results concerning the benefit were given.

An incremental SAT engine was proposed in [9]. In this work, the SAT solver is never released and conflict clauses are shared between subsequent similar SAT instances. This concept was applied for stuck-at test generation in [10]. Furthermore, a circuit-based learning strategy based on stuck-at fault partitioning was proposed. However, the use of fault dropping in industrial practice decreases the benefit significantly. Moreover, the computational overhead caused by the use of an external database restricts the amount of learned information.

The reuse of learned information for SAT-based ATPG for Path Delay Faults (PDF) was presented in [11]. However, the reuse is based on the time consuming task of unsatisfiable core extraction to identify path segments which cannot sensitized together.

\subsection{Contribution}

In this paper, we consider the problem of delay test generation and present efficient techniques to prune the search space by information learned during ATPG for previous faults. Due to a tight integration into a SAT-based ATPG algorithm, dynamically learned conflict clauses can be efficiently passed from one target fault to another and, by this, reduce the number of unclassified faults significantly. This approach works in an incremental manner but differs from incremental SAT, because subsequent SAT instances do not have to be similar to exploit the advantages.

Keeping all learned information causes a large memory overhead. Therefore, different strategies to identify unimportant clauses are presented to achieve a good tradeoff between the memory consumption and the number of unclassified faults. Furthermore, we propose the use of a Post-Classification Phase (PCP). In particular, in this phase, faults are classified which were aborted in the beginning of the ATPG run. Experimental results for transition delay and path delay test generation on large industrial circuits show the robustness and feasibility of the proposed approach.

The rest of the paper is structured as follows: Section 2 briefly presents the basics of SAT-based delay test generation and introduces pervasive conflict clauses. The efficient integration of dynamic learning is presented in Section 3. 
The concept of a PCP is proposed in Section 4. Section 5 introduces different dynamic learning strategies for experimental evaluation and Section 6 presents the experimental results. Conclusions are drawn in the last section.

\section{Preliminaries}

In Section 2.1, the most common delay fault models are introduced. Section 2.2 deals with the basics of SAT-based ATPG. The concept of pervasive conflict clauses is presented in Section 2.3.

\subsection{Delay Fault Models}

The most accurate delay fault model is the Path Delay Fault Model (PDFM) [12]. It captures small as well as large delay defects on a path from an input to an output.

The target of ATPG is to generate a test that initializes the desired transition and sensitizes the off-path inputs of $p$ according to a sensitization criterion. By this, the transition is propagated to an output (observation point). An off-path input is not located on $p$ but drives a gate $g$ which is located on $p$. Two time frames $t_{1}, t_{2}$ have to be considered during ATPG in order to initialize and launch a transition.

The sensitization criterion defines the quality of the generated test pattern. Test patterns generated with the non-robust sensitization criterion guarantee the detection of the PDF if no other delay fault is present. The robust sensitization criterion is superior to the non-robust sensitization criterion because it guarantees the detection of a delay fault if other delay faults occur in the circuit. Robust test patterns are harder to obtain than non-robust test patterns. For more details about sensitization criteria, we refer to [13].

The major drawback of the PDFM is the exponential number of paths in modern circuits. Testing all paths is therefore not applicable in practice. Usually, a small number of critical paths is extracted for ATPG.

The Transition Delay Fault Model (TDFM) [14] is less accurate than the PDFM. Here, the delay on a connection is assumed to be large enough that it can be observed along any sensitized sub-path from the fault site to an output. The number of faults is linear in the number of connections. Consequently, it provides a better fault coverage in practice than the PDFM and is therefore widely used in industry.

\subsection{SAT-based ATPG}

To apply a SAT solver to a circuit-oriented problem, e.g. ATPG, the problem is formulated as a Boolean formula in CNF. A CNF $\Phi$ in $m$ Boolean variables is a conjunction of $n$ clauses. Each clause is a disjunction of literals. A literal is a Boolean variable $(x)$ or its complement $(\bar{x})$. The CNF $\Phi$ is satisfied if all clauses are satisfied. A clause is satisfied if at least one literal of the clause is satisfied. The CNF $\Phi$ is said to be unsatisfiable iff no solution can be found that satisfies $\Phi$. The task of a SAT solver for a given $\Phi$ is to find a satisfying assignment or to prove that no such assignment exists.

In the following, the circuit-to-CNF conversion is briefly described. More information can be found in [15]. A Boolean variable is assigned to each connection in circuit $C$. The CNF $\Phi_{g}$ for each gate $g$ in $C$ is derived from the characteristic function which can be constructed using the truth table. The CNF $\Phi_{C}$ representing the circuit's function is then constructed by the conjunction of the CNFs of all gates $g_{1}, \ldots, g_{n} \in C$ :

$$
\Phi_{C}=\prod_{i=1}^{n} \Phi_{g_{i}}
$$

If two time frames have to be considered, two Boolean variables - one for each time frame - have to be assigned to each connection and the CNF for each gate has to be extracted for both time frames. Here, the variables of the corresponding time frame have to be used. This procedure and the correct modeling of flipflops is described in detail in [16]. If additional values, e.g. unknown or static values, are needed, a multiple-valued logic and a corresponding Boolean encoding have to be used; for further details see e.g. [6], [17].

The $\Phi_{C}$ has to be extended by the fault-specific constraints $\Phi_{F}$ for generating a test for fault $F$. Only a small necessary part of the circuit has to be included in the CNF $\left(\Phi_{C}^{F}\right)$ for reasons of efficiency. For example, for the PDFM, only the support of the output of the path is contained in $\Phi_{C}^{F}$. More formally, a test for $F$ is generated by evaluating the following formula:

$$
\Phi_{\text {test }}^{F}=\Phi_{C}^{F} \cdot \Phi_{F}
$$

\subsection{Pervasive Conflict Clauses}

The generation of additional implications, i.e. learning, to speed up ATPG was first proposed in [2] and have been improved continuously thereafter, e.g. in [18]. State-of-theart SAT solvers, e.g. [7], [8], [19], [20], employ an inherit learning feature. They benefit significantly from the use of conflict clauses. If a conflict occurs during the search, the conflict is analyzed and a conflict clause is generated, i.e. learning is performed. In a circuit-oriented problem, a conflict clause corresponds to a conflicting value assignment of connections. The recorded conflicts can be used to derive additional implications efficiently.

Conflict clause generation is done by resolution of those clauses which are responsible for the conflict. Such a clause avoids that the SAT solver reenters this non-solution search space again. By this, large parts of the search space are pruned and the search is accelerated. A procedure to generate powerful conflict clauses is presented in detail in [21].

As stated in Section 2.2, the CNF is composed of two parts: the circuit part and the fault-specific part. Conflict clauses which have their source only in clauses from the circuit part are fault independent and are called pervasive conflict clauses [4] (or pervasive clauses in the following). They can be reused in subsequent SAT instances to prune search space without running in the conflict first. On the other hand, conflict clauses which are generated using at least one clause from the fault-specific part are fault dependent. Those have to be discarded after solving the CNF.

\section{Integration of Dynamic Learning}

In this section, it is shown how dynamic learning can be efficiently integrated in SAT-based ATPG algorithms. An external database is used in [10]. The pervasive clauses are 
extracted after each run and transformed into a gate level representation. Each time a new SAT instance is built, each clause in the database is checked whether is can be reused for the current SAT instance. If it can be reused, the clause is transformed from the gate level representation to CNF. The transformation steps and the check procedure took much run time and limited the amount of learned information. In contrast, we propose the use of an internal database in combination with an efficient watch-list strategy to overcome this disadvantage.

Crucial for an efficient transfer of pervasive clauses is a permanent variable assignment to connections ${ }^{1}$. Due to a permanent variable assignment, all pervasive clauses can be stored in the internal database "as is". Thus, expensive transformation steps are avoided. Newly learned clauses can be stored efficiently inside the SAT solver by using pointers.

\subsection{Pervasive Conflict Clause Identification}

In this section, it is described how pervasive clauses can be identified. When building the SAT instance for fault $F$, it is easily possible to distinguish between fault independent clauses $\left(\Phi_{C}^{F}\right)$ and fault dependent clauses $\left(\Phi_{F}\right)$. All learned clauses that have their source in at least one fault dependent clause have to be discarded and cannot generally be reused for subsequent SAT instances. Two methods are used:

- ID identification - The variable with the highest ID is defined as a fixed upper limit $u$ after the permanent variable assignment has been done. If new variables have to be defined for $\Phi_{F}$, e.g. describing faulty circuitry, only variables with an ID larger than $u$ are used. Thus, if a learned clause contains at least one variable with an ID larger than $u$, the clause is discarded.

- Literal identification - The literal $\lambda$ is added to each fault dependent clause $c$ that do not use only new - fault dependent - variables. The literal $\lambda$ is set to false by an incremental assumption [20] before each run. By this, $c$ keeps the original meaning $-\lambda$ is redundant. Because $\lambda$ is only used in one phase, each conflict clause derived from $c$ contains also $\lambda$ and can easily be identified.

In summary, each learned clause $c=\left(l_{1}+\ldots+l_{n}\right)$ is checked after each run and discarded if the following holds:

$$
\bigvee_{i=1}^{n}\left(\left(l_{i}=\lambda\right) \vee\left(\operatorname{ID}\left(l_{i}\right)>u\right)\right)
$$

Otherwise, $c$ is a pervasive clause.

\subsection{Variable-based Activation}

Not all stored clauses should be reused in each SAT instance. Subsequent SAT instances may target faults from other regions of the circuit. A pervasive clause learnt from one part of the circuit is useless if that part is not included in the current SAT instance. Due to the large number of pervasive clauses in industrial designs, checking each single clause is not feasible and may outweigh the benefit. Therefore, the concept of variable-based activation is introduced. Here, the internal database is modeled as a watch-list (similar to the watch-list used for fast BCP presented in [7]).

1. However, this causes some overhead in the search algorithm of the SAT solver which can be adjusted by a slight modification.

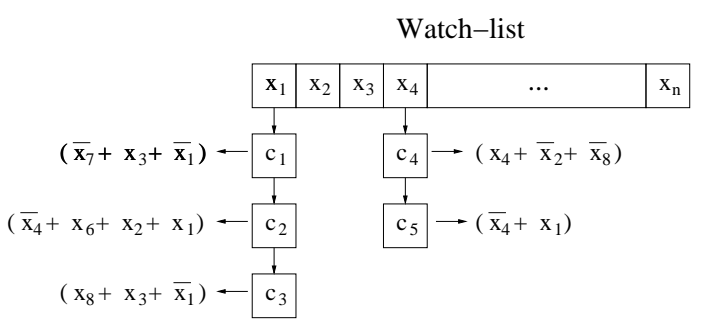

Figure 1. Illustration of a watch-list example

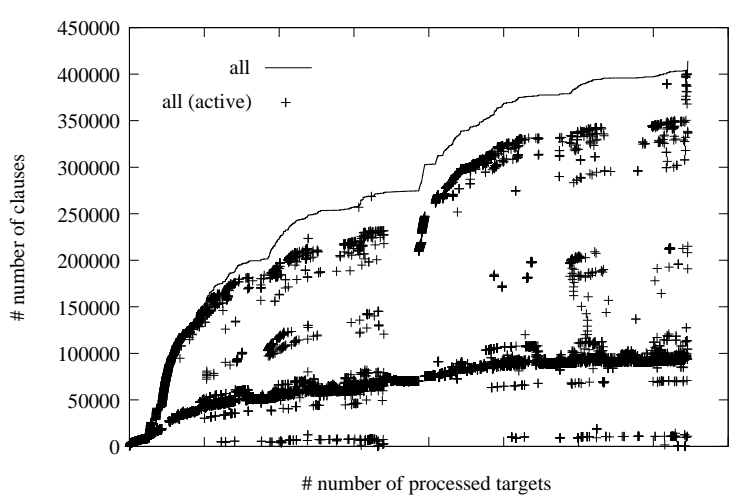

Figure 2. Progress of (active) learned clauses - p57k transition faults

A list of clauses is assigned to each variable $x_{i}$ of the circuit. These clauses are said to be "watched" by $x_{i}$. When a new pervasive clause $p$ is learned, one variable $x_{q}$ contained in $p$ is chosen. Then, $p$ is added to the list of $x_{q}$, i.e. $p$ is "watched" by $x_{q}$. In the following, the set of clauses which is watched by variable $x_{q}$ is denoted by $\omega\left(x_{q}\right)$. An example watch-list is illustrated in Figure 1. The variables are denoted by $x_{1}, \ldots, x_{n}$, and $c_{1}, \ldots, c_{5}$ are pervasive clauses.

By this, the set of pervasive clauses for $\Phi_{\text {test }}^{F}$ can be efficiently determined. All learned clauses which are "watched" by variables contained in $\Phi_{\text {test }}^{F}$ can be added directly to $\Phi_{\text {test }}^{F}$. In other words, when the $\operatorname{CNF} \Phi_{g}$ for gate $g$ with output variable $v_{g}$ is added to $\Phi_{\text {test }}^{F}$, the "watched" clause set $\omega\left(v_{g}\right)$ is added, too. The clauses of $\omega\left(v_{g}\right)$ are said to be "active" for $\Phi_{\text {test }}^{F}$. Those clauses which come from other parts of the circuits do not have to be considered and, therefore, cause no run time overhead. These clauses are "inactive" for $\Phi_{\text {test }}^{F}$.

However, a learned clause $p=\left(l_{1}+\ldots+l_{n}\right)$ is only useful for $\Phi_{\text {test }}^{F}$ if all variables are contained in $\Phi_{\text {test }}^{F}$. Otherwise, at least one variable remains unconstrained and can satisfy $p$. Nonetheless, it is sufficient to let a learned clause be "watched" by one variable. This is because the overhead of determining whether all variables are contained in $\Phi_{\text {test }}^{F}$ is higher than adding some "useless" clauses to $\Phi_{\text {test }}^{F}$.

\section{Post-Classification Phase}

In this section, the use of a post-classification phase is motivated and described. Consider the diagram shown in Figure 2. In this diagram, the progress of the number of learned clauses of ATPG for TFs for an industrial design is shown. On the abscissa, the progress in terms of targeted faults is denoted. The number of learned clauses is presented on the ordinate. The upper line shows the total number 
Table 1. Number of Learned Clauses

\begin{tabular}{|c|c|c|c|c|c|c|c|c|c|c|c|c|}
\hline & \multicolumn{6}{|c|}{ Path Delay Fault Model } & \multicolumn{6}{|c|}{ Transition Delay Fault Model } \\
\hline Circ & paths & all & Act $1 / 4$ & Act $1 / 2$ & DL 3 & DL 20 & targets & all & Act $1 / 4$ & Act $1 / 2$ & DL 3 & DL 20 \\
\hline b14 & 1,666 & 2,855 & 1,222 & 1,746 & 1,097 & 2,314 & 20,163 & 22,335 & 11,985 & 16,978 & 9,143 & 21,324 \\
\hline b15 & 3,696 & 1,862 & 1,193 & 1,777 & 890 & 1,803 & 25,087 & 25,349 & 10,831 & 17,553 & 4,730 & 18,555 \\
\hline b17 & 10,000 & 13,903 & 6,955 & 9,343 & 4,910 & 12,456 & 80,996 & 90,580 & 39,050 & 61,327 & 21,340 & 63,530 \\
\hline b18 & 10,000 & 18,217 & 10,187 & 14,465 & 6,921 & 17,786 & 301,012 & 670,081 & 315,590 & 536,267 & 103,238 & 367,080 \\
\hline $\mathrm{p} 44 \mathrm{k}$ & 10,000 & 7,576 & 8,274 & 8,770 & 7,925 & 7,214 & 65,484 & 93,077 & 56,478 & 73,486 & 31,475 & 92,567 \\
\hline p5 $57 \mathrm{k}$ & 10,000 & 816,963 & 505,244 & 663,183 & 83,921 & 485,227 & 14,921 & 414,227 & 180,443 & 287,797 & 51,456 & 232,409 \\
\hline p77k & 10,000 & 0 & 0 & 0 & 0 & 0 & 180,679 & 620,815 & 434,153 & 488,908 & 98,757 & 560,056 \\
\hline p80k & 10,000 & 587,137 & 252,765 & 400,175 & 73,913 & 369,813 & 16,729 & 120,645 & 47,636 & 71,298 & 24,550 & 98,252 \\
\hline $\mathrm{p} 88 \mathrm{k}$ & 10,000 & 61,267 & 33,013 & 46,976 & 22,469 & 60,838 & 56,024 & 63,340 & 35,504 & 48,397 & 22,861 & 55,609 \\
\hline $\mathrm{p} 99 \mathrm{k}$ & 10,000 & 119,056 & 50,751 & 77,790 & 17,267 & 86,254 & 41,668 & 292,777 & 97,156 & 173,223 & 34,603 & 171,128 \\
\hline $\mathrm{p} 177 \mathrm{k}$ & 10,000 & 129,261 & 66,103 & 91,973 & 28,251 & 120,386 & 99319 & $2,084,080$ & 904,505 & $1,355,935$ & 193,429 & 912,423 \\
\hline $\mathrm{p} 456 \mathrm{k}$ & 10,000 & 112,475 & 53,527 & 77,899 & 23,864 & 74,926 & 158,595 & $4,511,460$ & $2,548,415$ & $3,122,967$ & 436,570 & $1,623,043$ \\
\hline $\mathrm{p} 462 \mathrm{k}$ & 10,000 & 13,734 & 7,093 & 9,930 & 4,626 & 13,363 & 319,483 & 234,315 & 140,584 & 187,110 & 108,247 & 215,139 \\
\hline p565k & 10,000 & 169,865 & 74,965 & 122,033 & 14,454 & 100,210 & 107,787 & 930,337 & 568,341 & 741,698 & 185,040 & 583,180 \\
\hline $\mathrm{p} 1330 \mathrm{k}$ & 10,000 & 8,007 & 4,274 & 6,261 & 965 & 6,369 & 269,425 & 233,479 & 106,462 & 158,285 & 72,438 & 187,938 \\
\hline total & & $100 \%$ & $52.2 \%$ & $74.3 \%$ & $14.1 \%$ & $65.9 \%$ & & $100 \%$ & $53 \%$ & $70.5 \%$ & $13.4 \%$ & $50.0 \%$ \\
\hline
\end{tabular}

of learned clauses and the crosses presents the number of "active" clauses for each target fault.

The diagram clearly shows that faults targeted at the beginning of the ATPG run have access to a significant smaller number of learned clauses than faults targeted at the end of the ATPG run. Furthermore, our experiments have shown that - when using dynamic learning - most of the remaining unclassified faults appear at the beginning of the ATPG run. The ordering of the faults clearly has an influence on the classification. The effect can be mitigated by using a post-classification phase. The post-classification phase starts after all faults were targeted. In this phase, all faults that were aborted in the ATPG run, are targeted again. The benefit is that - in most cases - the search algorithm has access to significantly more learned clauses than in the previous ATPG call for these faults. Therefore, it is likely that many faults that were aborted in the previous phase can now be classified. Optionally, in this phase, the time limit can be decreased to save run time.

\section{Learning Strategies}

SAT solvers generate a large number of conflict clauses during their search. Keeping all pervasive clauses results in a large memory overhead. Furthermore, the efficiency of the $\mathrm{BCP}$ routine of the SAT solver can be decreased by too many clauses. The clause size was proposed as a selection criterion in [4]. Any clause that contains more literals than a userdefined maximum is discarded. In [10], the maximum size was set to only three literals. In this section, an additional selection criterion is introduced and the selection criteria chosen for experimental evaluation are presented.

State-of-the-art SAT solvers have an inherit feature to judge the importance of a learned conflict clause [19]. The importance of a learned clause is defined over the frequency of being involved in conflicts. Therefore, all learned conflict clauses have an activity value [19], which is increased each time a conflict clause is considered in the conflict analysis. Conflict clauses of lesser importance, i.e. with a low activity value, are discarded in intervals. This feature can be exploited as an additional selection criterion when using dynamic learning. The following strategies are chosen for experimental evaluation:

- all - After each run, all learned pervasive clauses are stored in the internal database and kept for subsequent
SAT instances.

- Act 1/4 - The learned pervasive clauses are ordered according to their activity values and the quarter having the highest activity value is stored in the internal database.

- Act 1/2 - The learned pervasive clauses are ordered according to their activity values and the half part having the highest activity value is stored.

- DL 3 - All learned pervasive clauses with at most three literals are kept for subsequent SAT instances (as done in [10]).

- DL 20 - All learned pervasive clauses with at most 20 literals $^{2}$ are kept for subsequent SAT instances.

\section{Experimental Results}

In this section, the experimental results for publicly available benchmarks (ITC'99) as well as for industrial circuits provided by NXP Semiconductors are presented. All experiments were carried out on a AMD 64-Bit Opteron (32768 MB, 2,8 GHz, GNU/Linux). A modified version of MiniSat v1.14 [20] was used as SAT solver.

\subsection{Dynamic Learning Strategies}

Experiments were performed for the PDFM (robust sensitization) and for the TDFM. The reader is referred to [16] and [17], respectively, for the concrete SAT modeling. A set of 10,000 paths with a length of at least 50 gates are chosen for PDF testing. At most 15 paths start from the same input to target different parts of the circuit. For the TDFM, all possible faults are targeted. Here, fault dropping is enabled during test generation. Table 1 shows the number of learned clauses for each strategy. The name of the circuit is given in column Circ. For the industrial circuits, the name denotes roughly their size. For example, p1330k contains over 1.3 million elements. Furthermore, the number of targeted paths (column paths) and targeted faults of strategy all (column targets) are presented. The average relative number of all learned clauses is given in the last row. It is notable that strategy $D L 3$ keeps only a small number of clauses compared to the other strategies. The reason why no learned clauses are kept for $\mathrm{p} 77 \mathrm{k}$ (PDFM) is that the untestability of each path was trivial to detect.

2. The number 20 was chosen due to the distribution of learned clause sizes in experimental evaluations. Preliminary studies have shown that this size is more beneficial than a size of 10 as used in [4]. 
Table 2. Experimental Results - Robust Path Delay Test Generation

\begin{tabular}{|c|c|c|c|c|c|c|c|c|c|c|c|c|c|c|c|c|c|}
\hline \multirow[b]{2}{*}{ Circ } & \multicolumn{2}{|c|}{ w/o DL } & \multicolumn{3}{|c|}{ all } & \multicolumn{3}{|c|}{ Act $1 / 4$} & \multicolumn{3}{|c|}{ Act $1 / 2$} & \multicolumn{3}{|c|}{ DL 3} & \multicolumn{3}{|c|}{ DL 20} \\
\hline & Ab. & Time & Mem & Ab. & Time & Mem & Ab. & Time & Mem & Ab. & Time & Mem & Ab. & Time & Mem & Ab. & Time \\
\hline b14 & 0 & $0: 50 \mathrm{~m}$ & $+3 \mathrm{M}$ & 0 & $0: 56 \mathrm{~m}$ & $+3 \mathrm{M}$ & 0 & $0: 57 \mathrm{~m}$ & $+3 \mathrm{M}$ & 0 & $0: 57 \mathrm{~m}$ & $+3 \mathrm{M}$ & 0 & $0: 58 \mathrm{~m}$ & $+3 \mathrm{M}$ & 0 & $0: 55 \mathrm{~m}$ \\
\hline b15 & 0 & $1: 52 \mathrm{~m}$ & $+0 \mathrm{M}$ & 0 & $1: 47 \mathrm{~m}$ & $+0 \mathrm{M}$ & 0 & $1: 55 \mathrm{~m}$ & $+0 \mathrm{M}$ & 0 & $1: 50 \mathrm{~m}$ & $+0 \mathrm{M}$ & 0 & $1: 49 \mathrm{~m}$ & $+3 \mathrm{M}$ & 0 & $1: 47 \mathrm{~m}$ \\
\hline b17 & 0 & $4: 47 \mathrm{~m}$ & $+2 \mathrm{M}$ & 0 & $5: 15 \mathrm{~m}$ & $+1 \mathrm{M}$ & 0 & $5: 23 \mathrm{~m}$ & +1 & 0 & $5: 16 \mathrm{~m}$ & M & 0 & $5: 21 \mathrm{~m}$ & M & 0 & $5: 13 \mathrm{~m}$ \\
\hline b18 & 6 & $5: 39$ & $+4 \mathrm{M}$ & 0 & $5: 54 \mathrm{~m}$ & $+3 \mathrm{M}$ & 0 & $6: 03 \mathrm{~m}$ & $+3 \mathrm{M}$ & 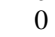 & $6: 01 \mathrm{~m}$ & $+2 \mathrm{M}$ & 0 & $5: 59 \mathrm{~m}$ & $+3 \mathrm{M}$ & 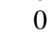 & $5: 57 \mathrm{~m}$ \\
\hline $\mathrm{p} 44 \mathrm{k}$ & 0 & $1: 20 \mathrm{~h}$ & $+0 \mathrm{M}$ & 0 & $20: 03 \mathrm{~m}$ & $+1 \mathrm{M}$ & 0 & $21: 30 \mathrm{~m}$ & $+1 \mathrm{M}$ & 0 & $20: 46 \mathrm{~m}$ & $+1 \mathrm{M}$ & 0 & $24: 56 \mathrm{~m}$ & $+0 \mathrm{M}$ & 0 & $19: 54 \mathrm{~m}$ \\
\hline p5 $57 \mathrm{k}$ & 39 & $49: 56 \mathrm{~m}$ & $159 \mathrm{M}$ & 93 & $1: 22 \mathrm{~h}$ & $+96 \mathrm{M}$ & 382 & $1: 14 \mathrm{~h}$ & $131 \mathrm{M}$ & 191 & $1: 19 \mathrm{~h}$ & $+9 \mathrm{M}$ & 927 & $56: 41 \mathrm{~m}$ & $+62 \mathrm{M}$ & 108 & $52: 08 \mathrm{~m}$ \\
\hline $\mathrm{p} 77 \mathrm{k}$ & 0 & $1: 00 \mathrm{~m}$ & $+0 \mathrm{M}$ & 0 & $1: 03 \mathrm{~m}$ & $+0 \mathrm{M}$ & 0 & $1: 03 \mathrm{~m}$ & $+0 \mathrm{M}$ & 0 & $1: 02 \mathrm{~m}$ & $+0 \mathrm{M}$ & 0 & $1: 03 \mathrm{~m}$ & $+0 \mathrm{M}$ & $\Omega$ & $1: 03 \mathrm{~m}$ \\
\hline $\mathrm{p} 80 \mathrm{k}$ & 547 & $17: 11 \mathrm{~m}$ & $+117 \mathrm{M}$ & 140 & $20: 08 \mathrm{~m}$ & $+40 \mathrm{M}$ & 248 & $17: 10 \mathrm{~m}$ & $+74 \mathrm{M}$ & 207 & $18: 23 \mathrm{~m}$ & $+8 \mathrm{M}$ & 335 & $16: 28 \mathrm{~m}$ & $+45 \mathrm{M}$ & 155 & $17: 36 \mathrm{~m}$ \\
\hline $\mathrm{p} 88 \mathrm{k}$ & 0 & $7: 58 \mathrm{~m}$ & $+7 \mathrm{M}$ & 0 & $6: 56 \mathrm{~m}$ & $+4 \mathrm{M}$ & . & $7: 17 \mathrm{~m}$ & $+5 \mathrm{M}$ & 0 & $7: 0$ & $+2 \mathrm{M}$ & 0 & $7: 05 \mathrm{~m}$ & $+7 \mathrm{M}$ & 0 & $6: 52 \mathrm{~m}$ \\
\hline p99k & 34 & $6 \mathrm{~m}$ & $+16 \mathrm{M}$ & 3 & $4: 26 \mathrm{~m}$ & $+5 \mathrm{M}$ & & $4: 22 \mathrm{~m}$ & $+9 \mathrm{M}$ & 3 & $4: 2$ & $+0 \mathrm{M}$ & 4 & $4: 2$ & +8 & 5 & $4: 21 \mathrm{~m}$ \\
\hline $\mathrm{p} 177 \mathrm{k}$ & 223 & $32: 2$ & M & 16 & $31: 25 \mathrm{~m}$ & $+16 \mathrm{M}$ & 79 & $37:$ & $+19 \mathrm{M}$ & 41 & $34:$ & +11 & 177 & 35: & +20 & 21 & $31: 29 \mathrm{~m}$ \\
\hline $56 \mathrm{k}$ & 171 & $30: 1$ & $+14 \mathrm{M}$ & 16 & $27: 16$ & $+6 \mathrm{M}$ & 27 & $27: 1$ & $+9 \mathrm{M}$ & 18 & 27: & & 35 & $28:$ & +6 & . & $26: 34 \mathrm{~m}$ \\
\hline $\mathrm{p} 462 \mathrm{k}$ & 0 & $8: 53 \mathrm{~m}$ & $+3 \mathrm{M}$ & 0 & $9: 10 \mathrm{~m}$ & $+2 \mathrm{M}$ & 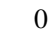 & $9: 12 \mathrm{~m}$ & $+2 \mathrm{M}$ & 0 & & $+2 \mathrm{M}$ & 0 & & $+3 \mathrm{M}$ & 0 & $9: 11 \mathrm{~m}$ \\
\hline p565k & 115 & $9: 56 \mathrm{~m}$ & $+32 \mathrm{M}$ & 31 & $9: 37 \mathrm{~m}$ & $+10 \mathrm{M}$ & 47 & $9: 26 \mathrm{~m}$ & $+20 \mathrm{M}$ & 41 & $9: 32 \mathrm{~m}$ & $+2 \mathrm{M}$ & 82 & $9: 31 \mathrm{~m}$ & $+10 \mathrm{M}$ & 32 & $9: 20 \mathrm{~m}$ \\
\hline p1330k & 0 & $19: 47 \mathrm{~m}$ & $2 \mathrm{M}$ & 0 & $20: 39 \mathrm{~m}$ & $+2 \mathrm{M}$ & 0 & $20: 39 \mathrm{~m}$ & $+2 \mathrm{M}$ & 0 & $21: 34 \mathrm{~m}$ & $+0 \mathrm{M}$ & 0 & $20: 47 \mathrm{~m}$ & $+2 \mathrm{M}$ & 0 & $20: 40 \mathrm{~m}$ \\
\hline total & 2635 & $4: 36 h$ & $+382 \mathrm{M}$ & 299 & $4: 06 \mathrm{~h}$ & $+189 \mathrm{M}$ & 788 & $4: 03 \mathrm{~h}$ & $+279 \mathrm{M}$ & 501 & $4: 06 h$ & $+44 \mathrm{M}$ & 1560 & $3: 46 h$ & $+173 \mathrm{M}$ & 328 & $3: 33 \mathrm{~h}$ \\
\hline
\end{tabular}

Table 2 reports the results for the PDF test generation. The results for the TDFM are shown in Table 3. The number of faults that could not be classified within the time interval of seven MiniSat restarts ${ }^{3}$ are presented in columns entitled $A b$. Columns named Time present the overall run time for ATPG. The memory overhead compared to the version without dynamic learning (column w/o DL) is shown in columns Mem. Time is given either in CPU minutes $(m)$ or CPU hours $(h)$. Memory overhead is presented in Megabyte $(M)$. The results are summed up for all circuits in the last row.

The results show that the number of unclassified faults can be significantly reduced by the proposed use of dynamic learning for both fault models. Keeping all learned clauses reduced the number of aborted faults for all circuits to only $11 \%$ for the PDFM and to only $0.8 \%$ for the TDFM.

Especially the reduction for the hard-to-test circuits p177k and $\mathrm{p} 456 \mathrm{k}$ (TDFM) is remarkable. However, the disadvantage of strategy all is the high memory consumption. The circuit p456k (TDFM) needs for example over 1GB of additional memory. In contrast, the amount of additional memory used by strategy $D L 3$ is negligible. However, the number of unclassified faults is increased by a factor of five (PDFM) and by a factor of 20 (TDFM) compared to strategy all. The activity-based strategies Act 1/4, Act 1/2 have less aborts than strategy $D L 3$, but they suffer also from their high memory consumption, especially for the TDFM. See for explanation the overall number of learned clauses presented in Table 1.

The best trade-off between the number of unclassified faults and the memory consumption can be achieved by strategy $D L 20$. Here, only $1.5 \%$ of the number of unclassified faults of $w / o D L$ remain unclassified for the TDFM. The number is - compared to strategy all - also only slightly increased for the PDFM (12\%). At the same time, the memory consumption of $D L 20$ can be significantly reduced. Although the number of unclassified faults is higher than for strategy Act 1/2 for the TDFM, strategy DL 20 should be preferred due to the smaller memory consumption. The run time can also be decreased as a side effect of this strategy.

In summary, the strategy $D L 20$ is the first choice for both delay fault models. If only few memory resources are available, a fair reduction can be achieved by using $D L 3$.

3. A restart is defined as a certain number of conflicts (100 at the beginning). After each restart, this number is increased (by $50 \%$ ).

\subsection{Post-Classification Phase}

The experimental results of the proposed postclassification phase are presented in Table 4. Due to page limitation, results are only reported for the strategies all and DL 20 and only the industrial circuits are considered. The post-classification phase is not applied for the ITC'99 benchmarks, because no aborts were produced. For comparison, column w/o $D L$ shows the number of unclassified faults without dynamic learning. Column entitled Time presents the additional time needed for the post-classification phase. The number of faults that were aborted in the previous phase but could be classified during the post-classification phase is given in column Diff. Column $A b$. shows the overall number of unclassified faults after the post-classification phase.

Roughly $4 \%$ of the ATPG run time is needed for the post-classification phase for all circuits for the PDFM. For the TDFM, the percentage is only $2 \%$ of the ATPG run time. About $80 \%$ of the remaining unclassified PDFs can be classified in this phase in total. For the TDFM, still $43 \%$ of the previously unclassified faults can additionally be classified. As a result, the number of unclassified PDFs. decreases to only $2.2 \%$ ( $D L 20: 2.8 \%)$ of those of w/o $D L$. The number of unclassified TDFs further decreases to only $0.5 \%$ ( $D L 20: 1.2 \%)$.In summary, the use of the post-classification phase can further reduce the number of aborted faults significantly. The run time overhead for the post-classification phase is negligible.

\section{Conclusions}

A high fault coverage for delay faults is needed to ensure that manufactured chips meet their timing specification. This requires robust ATPG algorithms producing only very few aborts. In this paper, we presented how dynamic learning techniques can be efficiently integrated into SATbased ATPG algorithms. Dynamically learned information can easily be passed to subsequent SAT instances by the proposed techniques. This prunes the search space for other target faults as well.

Different learning strategies were presented to reduce the high memory overhead. As a result, a good trade-off between run time, memory consumption and robustness of the ATPG algorithm is achieved. Experiments on publicly available benchmarks and on industrial circuits show that 
Table 3. Experimental Results - Transition Delay Test Generation

\begin{tabular}{|c|c|c|c|c|c|c|c|c|c|c|c|c|c|c|c|c|c|}
\hline \multirow[b]{2}{*}{ Circ } & \multicolumn{2}{|c|}{ w/o DL } & \multicolumn{3}{|c|}{ all } & \multicolumn{3}{|c|}{ Act $1 / 4$} & \multicolumn{3}{|c|}{ Act $1 / 2$} & \multicolumn{3}{|c|}{ DL 3} & \multicolumn{3}{|c|}{ DL 20} \\
\hline & Ab. & Time & Mem & Ab. & Time & Mem & Ab. & Time & Mem & Ab. & Time & Mem & Ab. & Time & Mem & Ab. & Time \\
\hline b14 & 0 & $3: 03 \mathrm{~m}$ & $+2 \mathrm{M}$ & 0 & $2: 57 \mathrm{~m}$ & $+1 \mathrm{M}$ & 0 & $2: 46 \mathrm{~m}$ & $+2 \mathrm{M}$ & 0 & $2: 53 \mathrm{~m}$ & $+1 \mathrm{M}$ & 0 & $2: 52 \mathrm{~m}$ & $+2 \mathrm{M}$ & 0 & $2: 55 \mathrm{~m}$ \\
\hline b15 & 0 & $3: 56 \mathrm{~m}$ & $+4 \mathrm{M}$ & 0 & $3: 59 \mathrm{~m}$ & $+1 \mathrm{M}$ & 0 & $3: 43 \mathrm{~m}$ & $+2 \mathrm{M}$ & 0 & $3: 48 \mathrm{~m}$ & $+0 \mathrm{M}$ & 0 & $3: 38 \mathrm{~m}$ & $+2 \mathrm{M}$ & 0 & $3: 50 \mathrm{~m}$ \\
\hline b17 & 0 & $17: 52 \mathrm{~m}$ & $+15 \mathrm{M}$ & 0 & $16: 50 \mathrm{~m}$ & $+5 \mathrm{M}$ & 0 & $15: 52 \mathrm{~m}$ & $+9 \mathrm{M}$ & 0 & $16: 08 \mathrm{~m}$ & $+1 \mathrm{M}$ & 0 & $15: 56 \mathrm{~m}$ & $+6 \mathrm{M}$ & 0 & $16: 07 \mathrm{~m}$ \\
\hline b18 & 3 & $1: 53 \mathrm{~h}$ & $+132 \mathrm{M}$ & 0 & $2: 36 \mathrm{~h}$ & $+52 \mathrm{M}$ & 0 & $2: 07 \mathrm{~h}$ & $+101 \mathrm{M}$ & 0 & $2: 36 \mathrm{~h}$ & $+5 \mathrm{M}$ & 0 & $1: 48 \mathrm{~h}$ & $+37 \mathrm{M}$ & 0 & $2: 02 \mathrm{~h}$ \\
\hline $\mathrm{p} 44 \mathrm{k}$ & 59 & $5: 35 \mathrm{~h}$ & $+17 \mathrm{M}$ & 0 & $1: 22 \mathrm{~h}$ & $+9 \mathrm{M}$ & 0 & $1: 20 \mathrm{~h}$ & $+13 \mathrm{M}$ & 0 & $1: 20 \mathrm{~h}$ & $+1 \mathrm{M}$ & 2 & $3: 53 \mathrm{~h}$ & $+8 \mathrm{M}$ & 0 & $1: 29 \mathrm{~h}$ \\
\hline $\mathrm{p} 57 \mathrm{k}$ & 275 & $1: 03 \mathrm{~h}$ & $+79 \mathrm{M}$ & 6 & $41: 10 \mathrm{~m}$ & $+26 \mathrm{M}$ & 13 & $34: 41 \mathrm{~m}$ & $+49 \mathrm{M}$ & 10 & $37: 15 \mathrm{~m}$ & $+1 \mathrm{M}$ & 13 & $34: 18 \mathrm{~m}$ & $+21 \mathrm{M}$ & 11 & $33: 43 \mathrm{~m}$ \\
\hline p $77 k$ & 5421 & $1: 18 \mathrm{~h}$ & $+30 \mathrm{M}$ & 0 & $27: 49 \mathrm{~m}$ & $+3 \mathrm{M}$ & 0 & $27: 39 \mathrm{~m}$ & $+9 \mathrm{M}$ & 0 & $26: 46 \mathrm{~m}$ & $+22 \mathrm{M}$ & 6690 & $1: 57 \mathrm{~h}$ & $+18 \mathrm{M}$ & 0 & $26: 58 \mathrm{~m}$ \\
\hline $\mathrm{p} 80 \mathrm{k}$ & 30 & $11: 26 \mathrm{~m}$ & $+17 \mathrm{M}$ & 4 & $7: 48 \mathrm{~m}$ & $+4 \mathrm{M}$ & 4 & $8: 10 \mathrm{~m}$ & $+8 \mathrm{M}$ & 6 & $7: 52 \mathrm{~m}$ & $-2 \mathrm{M}$ & 6 & $7: 57 \mathrm{~m}$ & $+9 \mathrm{M}$ & 6 & $7: 45 \mathrm{~m}$ \\
\hline $\mathrm{p} 88 \mathrm{k}$ & 0 & $34: 02 \mathrm{~m}$ & $+6 \mathrm{M}$ & 0 & $27: 13 \mathrm{~m}$ & $+2 \mathrm{M}$ & 0 & $26: 15 \mathrm{~m}$ & $+3 \mathrm{M}$ & 0 & $26: 26 \mathrm{~m}$ & $+0 \mathrm{M}$ & 0 & $26: 47 \mathrm{~m}$ & $+4 \mathrm{M}$ & 0 & $27: 04 \mathrm{~m}$ \\
\hline p99k & 81 & $22: 41 \mathrm{~m}$ & $+49 \mathrm{M}$ & 9 & $16: 39 \mathrm{~m}$ & $+10 \mathrm{M}$ & 11 & $15: 05 \mathrm{~m}$ & $+27 \mathrm{M}$ & 13 & $15: 46 \mathrm{~m}$ & $+1 \mathrm{M}$ & 36 & 18:06m & $+16 \mathrm{M}$ & 32 & $15: 46 \mathrm{~m}$ \\
\hline $\mathrm{p} 177 \mathrm{k}$ & 23422 & $85: 26 h$ & $+556 \mathrm{M}$ & 14 & $38: 03 \mathrm{~h}$ & $+217 \mathrm{M}$ & 48 & $24: 08 \mathrm{~h}$ & $+338 \mathrm{M}$ & 22 & $27: 56 \mathrm{~h}$ & $+11 \mathrm{M}$ & 1652 & $32: 23 h$ & $+92 \mathrm{M}$ & 241 & $21: 59 \mathrm{~h}$ \\
\hline $\mathrm{p} 456 \mathrm{k}$ & 35970 & $16: 12 \mathrm{~h}$ & $+1184 \mathrm{M}$ & 488 & $19: 17 \mathrm{~h}$ & $+611 \mathrm{M}$ & 1049 & $15: 52 \mathrm{~h}$ & $+821 \mathrm{M}$ & 516 & $15: 28 \mathrm{~h}$ & $-1 \mathrm{M}$ & 2120 & $7: 59 \mathrm{~h}$ & $+135 \mathrm{M}$ & 721 & $8: 34 h$ \\
\hline $\mathrm{p} 462 \mathrm{k}$ & 554 & $6: 26 h$ & $+9 \mathrm{M}$ & 2 & $5: 27 \mathrm{~h}$ & $+1 \mathrm{M}$ & 12 & $5: 36 \mathrm{~h}$ & $+5 \mathrm{M}$ & 9 & $5: 31 \mathrm{~h}$ & $-1 \mathrm{M}$ & 58 & $5: 44 h$ & $+4 \mathrm{M}$ & 3 & $5: 25 \mathrm{~h}$ \\
\hline p565k & 638 & $4: 45 \mathrm{~h}$ & $+140 \mathrm{M}$ & 14 & $4: 36 \mathrm{~h}$ & $+70 \mathrm{M}$ & 126 & $4: 45 \mathrm{~h}$ & $+101 \mathrm{M}$ & 52 & $4: 37 \mathrm{~h}$ & $+4 \mathrm{M}$ & 71 & $4: 32 \mathrm{~h}$ & $+43 \mathrm{M}$ & 12 & $4: 33 \mathrm{~h}$ \\
\hline p1330k & 3 & $10: 50 \mathrm{~h}$ & $+29 \mathrm{M}$ & 0 & $10: 29 \mathrm{~h}$ & $+10 \mathrm{M}$ & 0 & $10: 23 \mathrm{~h}$ & $+17 \mathrm{M}$ & 0 & $10: 26 \mathrm{~h}$ & $+4 \mathrm{M}$ & 3 & $10: 30 \mathrm{~h}$ & $+15 \mathrm{M}$ & 2 & $10: 32 \mathrm{~h}$ \\
\hline total & 66456 & $128: 23 \mathrm{~h}$ & $+2269 \mathrm{M}$ & 537 & $84: 15 \mathrm{~h}$ & $+1022 \mathrm{M}$ & 1263 & $66: 26 h$ & $+1505 \mathrm{M}$ & 628 & $70: 11 \mathrm{~h}$ & $+47 \mathrm{M}$ & 10651 & $70: 36 h$ & $+412 \mathrm{M}$ & 1028 & $56: 50 h$ \\
\hline
\end{tabular}

Table 4. Experimental Results - Post-Classification Phase

\begin{tabular}{|c|c|c|c|c|c|c|c|c|c|c|c|c|c|c|}
\hline \multirow[b]{3}{*}{ Circ } & \multicolumn{7}{|c|}{ Path Delay Fault Model } & \multicolumn{7}{|c|}{ Transition Delay Fault Model } \\
\hline & w/o DL & & & & & 20 & & w/o DL & & & & & 20 & \\
\hline & & Time & Diff & Ab. & Time & Diff & $\mathrm{Ab}$. & Ab. & Time & Diff & $\mathrm{Ab}$. & Time & Diff & $\mathrm{Ab}$. \\
\hline $\mathrm{p} 44 \mathrm{k}$ & 0 & & & 0 & & & $\Omega$ & 59 & & 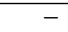 & 0 & & & \\
\hline & 39 & $0 \mathrm{~m}$ & 9 & 4 & $4 \mathrm{~m}$ & -99 & & 275 & $09 \mathrm{~m}$ & -1 & & $: 12 \mathrm{~m}$ & -2 & \\
\hline & 0 & & & 0 & & & & & & & & & & \\
\hline & 547 & & -98 & 42 & $3 \mathrm{~m}$ & 101 & 5 & 30 & $: 16 \mathrm{~m}$ & 0 & & $+0: 19 \mathrm{~m}$ & -2 & 4 \\
\hline & 0 & & & . & & & & 0 & & - & & & - & 0 \\
\hline & 3 & & -2 & & & -3 & & 81 & & -8 & & & -6 & 26 \\
\hline & & & -16 & 0 & & -21 & & & & -7 & & & -27 & 214 \\
\hline & 171 & & -15 & & $6 \mathrm{~m}$ & -6 & & & & -201 & 28 & & -200 & 521 \\
\hline & 0 & & 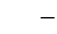 & 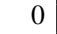 & & & c & 554 & & -1 & & & -2 & 1 \\
\hline & 11 & $26 \mathrm{~m}$ & -23 & 8 & $+0: 21 \mathrm{~m}$ & -25 & 7 & 638 & & -13 & & & -12 & \\
\hline 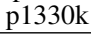 & 0 & & - & 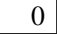 & - & 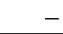 & 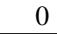 & 3 & & - & 0 & & -2 & 0 \\
\hline tota & 2629 & $+10: 27 \mathrm{~m}$ & -241 & 58 & $+8: 41 \mathrm{~m}$ & 255 & 73 & 66453 & $+1: 29 \mathrm{~h}$ & -231 & 306 & $+55: 38 \mathrm{~m}$ & -253 & 775 \\
\hline
\end{tabular}

the integration can reduce the number of aborts significantly. Therefore, the proposed approach is well suited to cope with the high delay fault coverage demands of the chip industry.

\section{Acknowledgment}

Parts of this research work were supported by the German Federal Ministry of Education and Research (BMBF) in the Project MAYA (01M3172B) and by the German Research Foundation (DFG) (DR 287/15-1).

\section{References}

[1] H. Fujiwara and T. Shimono, "On the acceleration of test generation algorithms," IEEE Trans. on Comp., vol. 32, no. 12, pp. 1137-1144, 1983.

[2] M. Schulz, E. Trischler, and T. Sarfert, "SOCRATES: A highly efficient automatic test pattern generation system," IEEE Trans. on Comp.-Aided Design for Circ. and Syst., vol. 7, no. 1, pp. 126-137, 1988.

[3] P. Stephan, R. Brayton, and A. Sangiovanni-Vincentelli, "Combinational test generation using satisfiability," IEEE Trans. on Comp.Aided Design for Circ. and Syst., vol. 15, pp. 1167-1176, 1996.

[4] J. Marques-Silva and K. Sakallah, "Robust search algorithms for test pattern generation," in Proc. Int'l Symp. on Fault-Tolerant Computing, 1997, pp. 152-157.

[5] E. Gizdarski and H. Fujiwara, "SPIRIT: A highly robust combinational test generation algorithm," IEEE Trans. on Comp.-Aided Design for Circ. and Syst., vol. 21, no. 12, pp. 1446-1458, 2002.

[6] R. Drechsler, S. Eggersglüß, G. Fey, A. Glowatz, F. Hapke, J. Schloeffel, and D. Tille, "On acceleration of SAT-based ATPG for industrial designs," IEEE Trans. on Comp.-Aided Design for Circ. and Syst., vol. 27, no. 7, pp. 1329-1333, 2008.

[7] M. Moskewicz, C. Madigan, Y. Zhao, L. Zhang, and S. Malik, "Chaff: Engineering an efficient SAT solver," in Proc. Design Automation Conf., 2001, pp. 530-535.

[8] J. Marques-Silva and K. Sakallah, "GRASP: A search algorithm for propositional satisfiability," IEEE Trans. on Comp., vol. 48, no. 5, pp. 506-521, 1999.
[9] J. Whittemore, J. Kim, and K. Sakallah, "SATIRE: A new incremental satisfiability engine," in Proc. Design Automation Conf., 2001, pp. 542-545.

[10] G. Fey, T. Warode, and R. Drechsler, "Reusing learned information in SAT-based ATPG," in Proc. Int'l Conf. on VLSI Design, 2007, pp. 69-76.

[11] K. Chandrasekar and M. S. Hsiao, "Integration of learning techniques into incremental satisfiability for efficient path-delay fault test generation," in Proc. Design, Automation and Test in Europe, 2005, pp. 1002-1007.

[12] G. Smith, "Model for delay faults based upon paths," in Proc. Int'l Test Conf., 1985, pp. 342-349.

[13] A. Krstić and K.-T. Cheng, Delay Fault Testing for VLSI Circ. Kluwer Academic Publishers, Boston, MA, 1998.

[14] J. Waicukauski, E. Lindbloom, B. Rosen, and V. Iyengar, "Transition fault simulation," Proc. IEEE Design \& Test of Comp., vol. 4, no. 2, pp. 32-38, 1987.

[15] T. Larrabee, "Test pattern generation using Boolean satisfiability," IEEE Trans. on Comp.-Aided Design for Circ. and Syst., vol. 11, no. 1, pp. 4-15, 1992.

[16] S. Eggersglüß, D. Tille, G. Fey, R. Drechsler, A. Glowatz, F. Hapke, and J. Schloeffel, "Experimental studies on SAT-based ATPG for gate delay faults," in Proc. Int'l Symp. on Multiple-Valued Logic, 2007.

[17] S. Eggersglüß, G. Fey, R. Drechsler, A. Glowatz, F. Hapke, and J. Schloeffel, "Combining multi-valued logics in SAT-based ATPG for path delay faults," in Proc. ACM \& IEEE Int'l Conf. on Formal Methods and Models for Codesign, 2007, pp. 181-187.

[18] W. Kunz and D. Pradhan, "Accelerated dynamic learning for test pattern generation in combinational circuits," IEEE Trans. on Comp.Aided Design for Circ. and Syst., vol. 12, no. 5, pp. 684-694, 1993.

[19] E. Goldberg and Y. Novikov, "BerkMin: a fast and robust SATsolver," in Proc. Design, Automation and Test in Europe, 2002, pp. 142-149.

[20] N. Eén and N. Sörensson, "An extensible SAT solver," in Proc. Int'l Conf. on Theory and Applications of Satisfiability Testing, vol. 2919, 2004, pp. 502-518.

[21] L. Zhang, C. Madigan, M. Moskewicz, and S. Malik, "Efficient conflict driven learning in a Boolean satisfiability solver," in Proc. Int'l Conf. on Comp.-Aided Design, 2001, pp. 279-285. 